Die Verschiebung des Kontextes bleibt freilich nicht ohne Rückwirkung. So kam es zu einer Entkopplung von sozialen Bewegungen, der Reduktion redaktioneller Aktivität auf das Erscheinen der Zeitung und zur Ausrichtung an den herrschenden Diskursen im Rechtssystem.

An diesem Punkt versuchen das seit 2006 bestehende HerausgeberInnenteam und die erneuerte Redaktion des juridikum (selbst-)kritisch anzusetzen. Ziel ist nicht die Revision der erreichten Verankerung in den juridischen Apparaten. Denn wenn man das Recht als relational autonom versteht, ${ }^{2}$ folgt daraus, dass die Interpretationskämpfe um die Qualifizierung als Recht/Unrecht - zumindest in letzter Konsequenz - in den Institutionen des Rechtssystems entschieden werden und daher auch in ihnen zu führen sind. Anspruch eines kritischen Zeitschriftenprojekts mit rechtlichem Schwerpunkt sollte daher die verstärkte Rückbindung an die Bedürfnisse sozialer Bewegungen außerhalb des Rechtssystems und deren Rückübersetzung in dieses sein.

Ein emanzipativer Anspruch impliziert auch die Auseinandersetzung mit der Form des Inhaltes. Diese hat auf Selbstermächtigung der Betroffenen zu zielen. Damit wird ein Beitrag dazu geleistet, die Verdinglichung durch Recht erkennbar zu machen: Recht kann als tagtäglich reproduziertes soziales Verhältnis entziffert werden, anstatt den Menschen ausschließlich als Objektivitätsüberhang entgegen zu treten.

Es braucht daher Strukturen der Reflexion und der Selbstvergewisserung. Die Etablierung einer lebendigen Redaktion, die sich über die Zusammenarbeit als intellektuell-praktisches Kollektiv konstituiert, erscheint daher als Notwendigkeit einer gegenhegemonialen Beteiligung in den Kämpfen um die Konstitution des Sozialen, dessen Teilaspekt das Recht bildet.

Dass (rechtliches) Wissen nur existiert, wenn es auch kommuniziert wird, bedeutet für ein fortschrittliches Zeitschriftenprojekt, dass sich die Arbeit nicht in der Produktion der Zeitung erschöpfen darf. Das juridikum versucht daher u.a. durch Heftpräsentationen, Seminare und Kooperationen das Heft zum Gegenstand der Auseinandersetzung zu machen. ${ }^{3}$

Wird das juridikum diesen Ansprüchen gerecht? Angesichts der »vorgefundenen, gegebenen und überlieferten Umstände« eigentlich ganz gut. Die Prekarisierung und Verdrängung kritischer Wissensproduktion in Zeiten des Neoliberalismus, in denen der Praxisbezug der Wissenschaften einzig nach ihrer Verwertbarkeit bemessen wird, haben die Rahmenbedingungen für ein solches Projekt nicht verbessert. Die Reflexion der Produktionsbedingungen und die Reartikulation von Ansprüchen an ein kritisches juristisches Projekt sind aber jedenfalls ein spannender Anfang.

\title{
3. Forum Recht Redaktion Forum Recht
}

Das Forum Recht wird seit 1983 vom Bundesarbeitskreis Kritischer Jura-Gruppen (bakj) und dem Forum Recht e.V. herausgegeben und erscheint einmal pro Quartal. Es versteht sich als rechtspolitisches Magazin, das kritische Rechtswis-

\footnotetext{
2 Sonja Buckel, Subjektivierung und Kohäsion - Zur Rekonstruktion einer materialistischen Theorie des Rechts, Weilerswist 2007.

3 Siehe dazu und für einen allgemeinen Überblick www.juridikum.at.
} 
senschaft betreiben will. Dies bedeutet, die gesellschaftlichen Machtverhältnisse aus dem Blickwinkel des Rechts zu beleuchten und zu hinterfragen, um einen Beitrag zu einer emanzipatorischen Politik zu liefern. Dieses zu Grunde liegende Verständnis von kritischer Rechtswissenschaft spiegelt sich im Anspruch, den regelmäßig bearbeiteten Themen-Feldern sowie der Organisations- und Arbeitsweise des Projektes wider.

\section{Übersetzungshilfe}

Forum Recht versteht sich gleich in doppelter Hinsicht als Dolmetscherin. Zum einen gilt es, die im Rechts-Code ausgetragenen Konflikte in den Bereich des Politischen zurück zu übersetzen. Recht ist vor allem eine Konflikt-Verschleierungs-Technik. Es gilt, den Schleier um diese Konflikte zu lüften, und Interessen, Intentionen und AkteurInnen zu benennen. Dabei ist es immer hilfreich, das Recht - abweichend vom Alltag an den juristischen Fakultäten - als soziales Phänomen und eben nicht bloß dogmatisch-hermeneutisch zu betrachten. Deshalb legt Forum Recht Wert darauf, ein interdisziplinäres Projekt zu sein, und veröffentlicht sehr gerne Beiträge aus sozialwissenschaftlichen Perspektiven. Dem Anspruch nach Entschleierung der rechtsförmig getarnten Interessengegensätze entsprechend, widmet sich Forum Recht häufig Themen-Feldern, bei denen diese Gegensätze besonders stark ausgeprägt sind. Klassischerweise sind das Menschen- und Bürgerrechte, Flüchtlings- und Asylpolitik oder feministische Rechtswissenschaft, aber auch Arbeits- und Sozialrecht, Umweltpolitik oder die Geschichte der NS-Justiz.

Zum anderen müssen juristische Debatten in eine wenig voraussetzungsreiche, klare Sprache übersetzt werden. Was in Forum Recht steht, soll für möglichst viele Menschen verständlich - ob Jura-Studierende oder nicht, ob im zweiten oder im fünfzehnten Semester - und auch konsumierbar sein. Diesen Anspruch sichert eine Redaktion, deren Mitglieder sich noch in der Ausbildung befinden, deren Sprachstil also noch nicht endgültig akademisch (de-)formiert ist. Wichtig ist auch die »Promi-Klausel«, die es etablierten RechtswissenschaftlerInnen verbietet, Texte in Forum Recht zu publizieren. Auch die Formvorgabe, dass Artikel maximal fünf Seiten Länge aufweisen dürfen, verhindert habituelle Ausschweifungen und zwingt zur Pointe. Schließlich dürfte Forum Recht diejenige juristische Zeitschrift sein, die am meisten Wert darauf legt, keine Bleiwüste zu sein. Stattdessen soll mit schickem Layout und verständlichen Texten, die auf den Punkt kommen, Auge und Geist gleichermaßen geschmeichelt werden.

\section{Talentschuppen}

Weiterhin versucht Forum Recht, (Jura-)Studierende durch das Zur-VerfügungStellen von Publikationsraum zu ermutigen, rechtskritische Beiträge zu verfassen. So können Studierende das Know-How erwerben, einerseits eine politische Analyse juristischer Vorgänge für die Linke (nicht verengt auf die Partei!) vorzunehmen und es ihr andererseits zu ermöglichen, sich deutlich und fundiert in die juristischen Debatten einzumischen. Solche Beiträge können, außer im jeweiligen Schwerpunkt einer Ausgabe - 2007 ging es beispielsweise um »Daten und Taten: Die Sicherheitsorgane langen $\mathrm{zu}$ «, »NS-Unrecht: Kontinuität und Gegenwart«, »Unheilige Allianz: Religion und Recht«, »Lebensformenzwang: Familie 
im Recht « - in den Rubriken »Forum « und »Ausbildung « veröffentlicht werden.

Um Studierende möglichst früh auf Forum Recht hinzuweisen, erscheint einmal jährlich ein Erstsemesterinfo für StudienanfängerInnen, das kostenlos an den Fakultäten verteilt wird.

Alles in allem versteht sich Forum Recht also gerade nicht als juristische Fachzeitschrift im engeren Sinne, sondern als rechtskritisches Magazin aus dem linken Spektrum: geschrieben für Uni und soziale Bewegung, in erster Linie von und für JuristInnen in der Ausbildung.

\section{Myops - Aufklärung durch Kritik Rainer Maria Kiesow}

\section{A. Lage (I)}

Der juristische Zeitschriftenmarkt wird von zwei Typen bestimmt:

- Die rechtsdogmatische Zeitschrift. Es handelt sich um juristische Zeitschriften im engeren Sinne, deren Fokus auf der Praxis (Rechtsanwälte, Richter, Unternehmens-/ Verbandsjuristen), der Ausbildung (Jurastudium) oder der Lehre (wissenschaftlich-dogmatische Diskussion/Fortbildung des Rechts) liegt. Beispiele: Neue Juristische Wochenschrift, Jura, Betriebs-Berater, Archiv für die civilistische Praxis. Diese Zeitschriften sind »betriebsnotwendig«.

- Die juristische Grundlagenzeitschrift. Es handelt sich um juristische Zeitschriften im weiteren Sinne, deren Fokus auf den historischen, sozialwissenschaftlichen, philosophischen, theoretischen, wenn man so will: kulturwissenschaftlichen Dimensionen des Rechts liegt. Beispiele: Die drei Sektionen der Savigny-Zeitschrift, Archiv für Rechts- und Sozialphilosophie, Zeitschrift für Rechtssoziologie, Rechtstheorie. Diese Zeitschriften sind aus je verschiedenen historischen Gründen entstanden und haben aus ebenso je verschiedenen historischen Gründen Höhen und Tiefen erlebt. Seit Jahren befinden sie sich geradezu ausnahmslos im Tief. Ihre Reflexionsaufgabe im Hinblick auf das Rechtssystem aufgebend, sind sie zu Abladestätten spezialistischer Forschung verkommen. Keine noch so abseitige und exaktheitheischende Frage wird ausgelassen, und die Antwort wird gedruckt. Das mag einschläfernd sein, ist jedoch nicht zu beanstanden. Auch die kleinste Provinz, der Rechtsgeschichte etwa, darf, kann und soll beackert werden. Es kann aber kaum übersehen werden, dass mit der Abnahme der Bedeutung der juristischen Grundlagenfächer auch deren Publikationsorgane unbedeutend werden. Selbst die Kritische Vierteljahresschrift für Gesetzgebung und Rechtswissenschaft ist nicht mehr der selbstverständliche Ort allerlebendigster Debatten. Und die Kritische Justiz?

Nun, die KJ ist sicher nach wie vor ein must für den die Rechtslage hinterfragenden Juristen. Die KJ ist das traditionsreiche Kampfschiff einer kritischen Reflexion von Recht und Gesellschaft. Nur: »Was ist Kritik?« - um den Titel eines berühmten Vortrags von Michel Foucault aufzunehmen, in dem Kants Aufklärungsfrage abgehandelt wird. Also, was ist Kritik heute? Früher war die Sache noch einfach. Die bisherigen kritischen Unternehmungen in der juristischen Publikationslandschaft, mit der KJ als Speerspitze, waren links, und es war dieser politische Standpunkt, der die Kritik befeuerte und sie schlagend machte. Doch, 